\title{
Corresponding-states model for the correlation and prediction of the surface tension of silanes
}

\author{
Shangguo Yang $\dagger^{\dagger}$, Jianxiang Tian $\dagger^{\dagger *} \#$, Hua Jiang $\S$, \\ A. Mulero"* , I. Cachadiñaף \\ †Department of Physics, Qufu Normal University, Qufu 273165, P. R. China

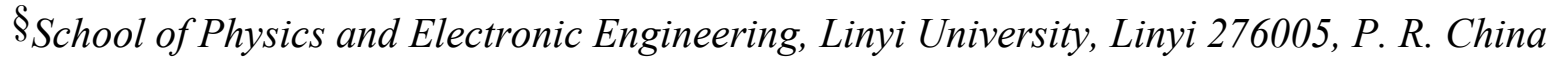 \\ \#Department of Physics, Dalian University of Technology, Dalian 116024, P. R. China \\ ¿Department of Applied Physics, University of Extremadura, Badajoz 06006, Spain \\ *Corresponding authors \\ jxtian@dlut.edu.cn \\ mulero@unex.es
}

\section{Supporting Information}

Tables S1 and S2, as well as Figures S1 to S5 are included. Table S1 contains the details of the selected data as well as the obtained AAD when DCP model is used. Table S2 contains the values of the physical properties of fluids used as inputs in the different correlation models. Figure S1 shows the results for Decamethylcyclopentasiloxane. Figure S2 shows the values for PD given by Eq. (14) for all the considered surface tension values. Figures S3 and S4 shows the results for Dimethyldimethoxysilane and Methyl Silane, respectively. Figure S5 shows the PDs for Ethyltrichlorosilane. 
Table S1. Summary of collected data (P: predicted; S: smoothed; E: experimental), temperature range and AADs obtained originally by Di Nicola et al. (Ref. 55 in the main text) and obtained by using the same DCP model for the new considered data.

\begin{tabular}{|c|c|c|c|c|c|c|c|}
\hline \multirow[b]{2}{*}{ Silane name } & \multicolumn{3}{|c|}{ Number of point } & \multirow[b]{2}{*}{$T_{\min }$} & \multirow[b]{2}{*}{$T_{\max }$} & \multirow{2}{*}{$\begin{array}{c}\text { AAD } \\
\text { Eq. (5) for } \\
\text { new } \\
\text { considered } \\
\text { data }\end{array}$} & \multirow{2}{*}{$\begin{array}{c}\text { AAD } \\
\begin{array}{c}\text { Eq. (5) from } \\
\text { original }\end{array} \\
\text { results by Di } \\
\text { Nicola } \text { et al. } \\
\text { (Ref. } 55 \text { in } \\
\text { the main text) }\end{array}$} \\
\hline & $\mathrm{E}$ & $\mathrm{S}$ & $\mathrm{P}$ & & & & \\
\hline Dimethyldichlorosilane & 7 & 0 & 0 & 273.15 & 333.15 & 2.52 & 1.4 \\
\hline Diphenyldichlorosilane & 7 & 0 & 0 & 303.15 & 413.15 & 2.55 & 3.7 \\
\hline Methyl Dichlorosilane & 9 & 0 & 0 & 268.15 & 308.15 & 0.93 & 1.0 \\
\hline Methyl Trichlorosilane & 14 & 0 & 0 & 273.15 & 393.15 & 0.74 & 1.4 \\
\hline Tetrachlorosilane & 11 & 0 & 0 & 273.15 & 328.15 & 4.24 & 1.9 \\
\hline Trichlorosilane & 21 & 0 & 0 & 263.15 & 313.15 & 3.54 & 2.4 \\
\hline Trimethylchlorosilane & 0 & 0 & 9 & 273.15 & 323.15 & 3.65 & 2.2 \\
\hline Decamethylcyclopentasiloxane & 28 & 0 & 0 & 229.15 & 555.66 & 4.08 & 4.0 \\
\hline Dodecamethylcyclohexasiloxane & 23 & 0 & 0 & 270.15 & 581.22 & 8.37 & 7.4 \\
\hline Hexamethylcyclotrisiloxane & 25 & 0 & 0 & 337.15 & 498.78 & 1.15 & 2.2 \\
\hline Octamethylcyclotetrasiloxane & 28 & 0 & 0 & 290.73 & 527.85 & 4.18 & 2.8 \\
\hline Decamethyltetrasiloxane & 13 & 0 & 0 & 293.15 & 333 & 2.53 & 3.5 \\
\hline Dodecamethylpentasiloxane & 11 & 0 & 0 & 293.15 & 328.15 & 3.67 & 2.7 \\
\hline Eicosamethylnonasiloxane & 17 & 0 & 0 & 202.15 & 636.48 & 5.22 & 4.8 \\
\hline Octadecamethyloctasiloxane & 8 & 0 & 0 & 298.15 & 337.15 & 11.32 & 10.3 \\
\hline Octamethyltrisiloxane & 11 & 0 & 0 & 293.15 & 328.15 & 0.60 & 1.5 \\
\hline Tetradecamethylhexasiloxane & 5 & 0 & 0 & 293.15 & 328.15 & 5.55 & 4.6 \\
\hline Dimethyldimethoxysilane & 12 & 0 & 0 & 193 & 471.6 & 22.99 & 21.8 \\
\hline Tetraethoxysilane & 6 & 0 & 0 & 289.65 & 333.65 & 13.08 & 13.3 \\
\hline Hexamethyldisilazane & 10 & 0 & 0 & 298.15 & 380 & 2.54 & 3.7 \\
\hline Silane & 0 & 9 & 0 & 88.5 & 161.3 & 0.36 & 1.1 \\
\hline Tetraethyl Silane & 11 & 0 & 0 & 288.15 & 398.65 & 2.57 & 3.6 \\
\hline Gamma-aminopropyltriethoxysilane & 0 & 0 & 16 & 140 & 571.14 & 26.28 & 25.5 \\
\hline 3-chloropropyltrichlorosilane & 0 & 0 & 16 & 224.3 & 594.99 & 2.45 & - \\
\hline (3-methylacryloxypropyl)trichlorosilane & 0 & 0 & 15 & 220 & 637.29 & 2.66 & - \\
\hline Dichlorosilane & 0 & 0 & 10 & 173 & 413 & 5.20 & - \\
\hline Ethyltrichlorosilane & 0 & 0 & 16 & 167.55 & 503.96 & 7.93 & - \\
\hline Methyl Chlorosilane & 0 & 0 & 10 & 139.05 & 397.8 & 15.58 & - \\
\hline
\end{tabular}




\begin{tabular}{|l|c|c|c|c|c|c|c|}
\hline Phenylmethyldichlorosilane & 0 & 0 & 9 & 229.65 & 542.01 & 10.42 & - \\
\hline Hexadecamethylcyclooctasiloxane & 0 & 0 & 16 & 304.7 & 620.28 & 15.20 & - \\
\hline [3-(2,3-epoxyproxy)propyl]trimethoxysilane & 0 & 0 & 12 & 160.75 & 618.22 & 30.89 & - \\
\hline [3-(mercapto)propyl]triethoxysilane & 0 & 0 & 15 & 210 & 582.75 & 10.87 & - \\
\hline Bis[3-(triethoxysilyl)propyl]disulfide & 0 & 0 & 16 & 270 & 810.27 & 3.88 & - \\
\hline Bis[3-trimethoxysilyl)propyl]disulfide & 0 & 0 & 16 & 250 & 662.58 & 20.56 & - \\
\hline Trimethoxysilane & 0 & 0 & 11 & 159.6 & 472.5 & 4.09 & - \\
\hline Methyl Silane & 0 & 0 & 11 & 116.34 & 317.25 & 31.70 & - \\
\hline
\end{tabular}

Table S2. Physical properties of fluids, $T$ is in kelvin, $V_{c}$ is in $\mathrm{m}^{3} / \mathrm{kmol}, \rho_{c}$ is in $\mathrm{mol} / \mathrm{l}$, and $P_{c}$ is in $b a r$. Data from DIPPR database (Ref. 56 in the main text).

\begin{tabular}{|c|c|c|c|c|c|c|c|c|c|c|}
\hline Silane name & $T_{t r}$ & $T_{c}$ & $T_{b}$ & $T_{\min }$ & $\sigma_{\max }$ & $G r$ & $\rho_{c}$ & $P_{c}$ & $V_{c}$ & $\omega$ \\
\hline Decamethylcyclopentasiloxane & 229.15 & 617.4 & 484.1 & 229.15 & 0.023 & $6.37 \mathrm{E}-10$ & 0.813008 & 10.35 & 1.23 & 0.622268 \\
\hline Dodecamethylcyclohexasiloxane & 270.15 & 645.8 & 518.15 & 270.15 & 0.021 & 7.24E-10 & 0.675676 & 9.005 & 1.48 & 0.709522 \\
\hline Hexamethylcyclotrisiloxane & 337.15 & 554.2 & 407.15 & 337.15 & 0.014351 & $4.92 \mathrm{E}-10$ & 1.44509 & 17.8 & 0.692 & 0.496173 \\
\hline Octamethylcyclotetrasiloxane & 290.73 & 586.5 & 448.15 & 290.73 & 0.0184 & $5.83 \mathrm{E}-10$ & 1.01626 & 13.172 & 0.984 & 0.583737 \\
\hline Eicosamethylnonasiloxane & 202.15 & 707.2 & 606.4 & 202.15 & 0.025514 & $1.23 \mathrm{E}-09$ & 0.409836 & 5.75 & 2.44 & 1.05 \\
\hline Dimethyldichlorosilane & 197.05 & 520.35 & 343.35 & 273.15 & 0.02229 & $3.54 \mathrm{E}-10$ & 2.7933 & 34.9 & 0.358 & 0.26747 \\
\hline Diphenyldichlorosilane & 251.15 & 814 & 577.25 & 303.15 & 0.03724 & $5.86 \mathrm{E}-10$ & 1.48368 & 23.3 & 0.674 & 0.42829 \\
\hline Methyl Dichlorosilane & 182.55 & 483 & 314.7 & 268.15 & 0.02212 & $3.26 \mathrm{E}-10$ & 3.46021 & 39.5 & 0.289 & 0.275755 \\
\hline Methyl Trichlorosilane & 195.35 & 517 & 339.55 & 273.15 & 0.02246 & $3.84 \mathrm{E}-10$ & 2.94118 & 35.3 & 0.34 & 0.263392 \\
\hline Tetrachlorosilane & 204.3 & 507 & 330 & 273.15 & 0.02126 & $3.77 \mathrm{E}-10$ & 3.06748 & 35.9 & 0.326 & 0.231765 \\
\hline Trichlorosilane & 144.95 & 479 & 305 & 263.15 & 0.02109 & $3.44 \mathrm{E}-10$ & 3.73134 & 41.7 & 0.268 & 0.203076 \\
\hline Trimethylchlorosilane & 215.45 & 497.75 & 330.75 & 273.15 & 0.01961 & $3.44 \mathrm{E}-10$ & 2.73224 & 32 & 0.366 & 0.270122 \\
\hline Decamethyltetrasiloxane & 205.15 & 599.4 & 467.55 & 293.15 & 0.0176 & $6.83 \mathrm{E}-10$ & 0.909091 & 11.9 & 1.1 & 0.659628 \\
\hline Dodecamethylpentasiloxane & 192 & 628.4 & 503.1 & 293.15 & 0.0181 & $7.31 \mathrm{E}-10$ & 0.714286 & 9.45 & 1.4 & 0.720156 \\
\hline Octadecamethyloctasiloxane & 210.15 & 688.9 & 583.6 & 298.15 & 0.01878 & $1.14 \mathrm{E}-09$ & 0.405515 & 6.2416 & 2.466 & 0.983675 \\
\hline Octamethyltrisiloxane & 193.15 & 564.4 & 425.7 & 293.15 & 0.0173 & $5.74 \mathrm{E}-10$ & 1.15207 & 14.6 & 0.868 & 0.537728 \\
\hline Tetradecamethylhexasiloxane & 214.15 & 653.2 & 532.95 & 293.15 & 0.01845 & $8.70 \mathrm{E}-10$ & 0.595238 & 8.0351 & 1.68 & 0.776908 \\
\hline Dimethyldimethoxysilane & 193 & 524 & 354.55 & 193 & 0.034531 & $3.79 \mathrm{E}-10$ & 2.26757 & 26.6 & 0.441 & 0.262922 \\
\hline Tetraethoxysilane & 190.97 & 592.2 & 440.9 & 289.65 & 0.02221 & $5.46 \mathrm{E}-10$ & 1.55039 & 20.45 & 0.645 & 0.632531 \\
\hline Hexamethyldisilazane & 0 & 544 & 399.15 & 298.15 & 0.01816 & $5.420 \mathrm{E}-10^{\mathrm{a}}$ & 1.63132 & 19.2 & 0.613 & 0.5101 \\
\hline Silane & 88.48 & 269.7 & 161 & 88.5 & 0.02622 & $1.07 \mathrm{E}-10$ & 7.5358 & 48.4 & 0.1327 & 0.093755 \\
\hline Tetraethyl Silane & 190.65 & 606 & 426.56 & 288.15 & 0.0236 & $5.251 \mathrm{E}-10^{\mathrm{a}}$ & 1.70358 & 24 & 0.587 & 0.400223 \\
\hline Gamma-aminopropyltriethoxysilane & 140 & 634.6 & 493.05 & 140 & 0.064966 & $5.94 \mathrm{E}-10$ & 1.25156 & 19.11 & 0.799 & 0.889544 \\
\hline 3-chloropropyltrichlorosilane & 224.3 & 661.1 & 455.45 & 224.3 & 0.038929 & $5.30 \mathrm{E}-10$ & 2.04918 & 29.27 & 0.488 & 0.381075 \\
\hline (3-methylacryloxypropyl)trichlorosila & 220 & 708.1 & 523.8 & 220 & 0.043808 & $6.47 \mathrm{E}-10$ & 1.50602 & 21.13 & 0.664 & 0.626713 \\
\hline
\end{tabular}




\begin{tabular}{|c|c|c|c|c|c|c|c|c|c|c|}
\hline ne & & & & & & & & & & \\
\hline Dichlorosilane & 151.15 & 451.5 & 281.45 & 173 & 0.030317 & $2.71 \mathrm{E}-10$ & 4.21941 & 45.99 & 0.237 & 0.163 \\
\hline Ethyltrichlorosilane & 167.55 & 559.95 & 371.05 & 167.55 & 0.041608 & $4.01 \mathrm{E}-10$ & 2.48139 & 33.3 & 0.403 & 0.269778 \\
\hline Methyl Chlorosilane & 139.05 & 442 & 281.85 & 139.05 & 0.030174 & $2.58 \mathrm{E}-10$ & 4.06504 & 41.7 & 0.246 & 0.225204 \\
\hline Phenylmethyldichlorosilane & 229.65 & 689 & 477.35 & 229.65 & 0.040412 & $4.83 \mathrm{E}-10$ & 1.9685 & 28.7 & 0.508 & 0.402222 \\
\hline Hexadecamethylcyclooctasiloxane & 304.7 & 689.2 & 576.35 & 304.7 & 0.02042 & $8.60 \mathrm{E}-10$ & 0.505051 & 6.92 & 1.98 & 0.882565 \\
\hline $\begin{array}{l}\text { [3-(2,3-epoxyproxy)propyl]trimethoxy } \\
\text { silane }\end{array}$ & 160.75 & 701.4 & 535 & 160.75 & 0.075 & $6.64 \mathrm{E}-10$ & 1.36612 & 18.12 & 0.732 & 0.760454 \\
\hline [3-(mercapto)propyl]triethoxysilane & 210 & 647.5 & 498.8 & 210 & 0.042685 & $6.16 \mathrm{E}-10$ & 1.3587 & 18.63 & 0.736 & 0.836974 \\
\hline Bis[3-(triethoxysilyl)propyl]disulfide & 270 & 900.3 & 731.4 & 270 & 0.036952 & $9.73 \mathrm{E}-10$ & 0.666667 & 10.22 & 1.5 & 0.888792 \\
\hline $\begin{array}{l}\text { Bis[3-(trimethoxysilyl)propyl]disulfid } \\
\text { e }\end{array}$ & 250 & 736.2 & 599.5 & 250 & 0.047099 & $9.22 \mathrm{E}-10$ & 0.900901 & 13.09 & 1.11 & 1.15507 \\
\hline Trimethoxysilane & 159.6 & 525 & 357.5 & 159.6 & 0.03648 & $3.95 \mathrm{E}-10$ & 2.45098 & 28.9 & 0.408 & 0.322392 \\
\hline Methyl Silane & 116.34 & 352.5 & 216.25 & 116.34 & 0.021274 & $1.91 \mathrm{E}-10$ & 4.87805 & 47 & 0.205 & 0.131449 \\
\hline
\end{tabular}




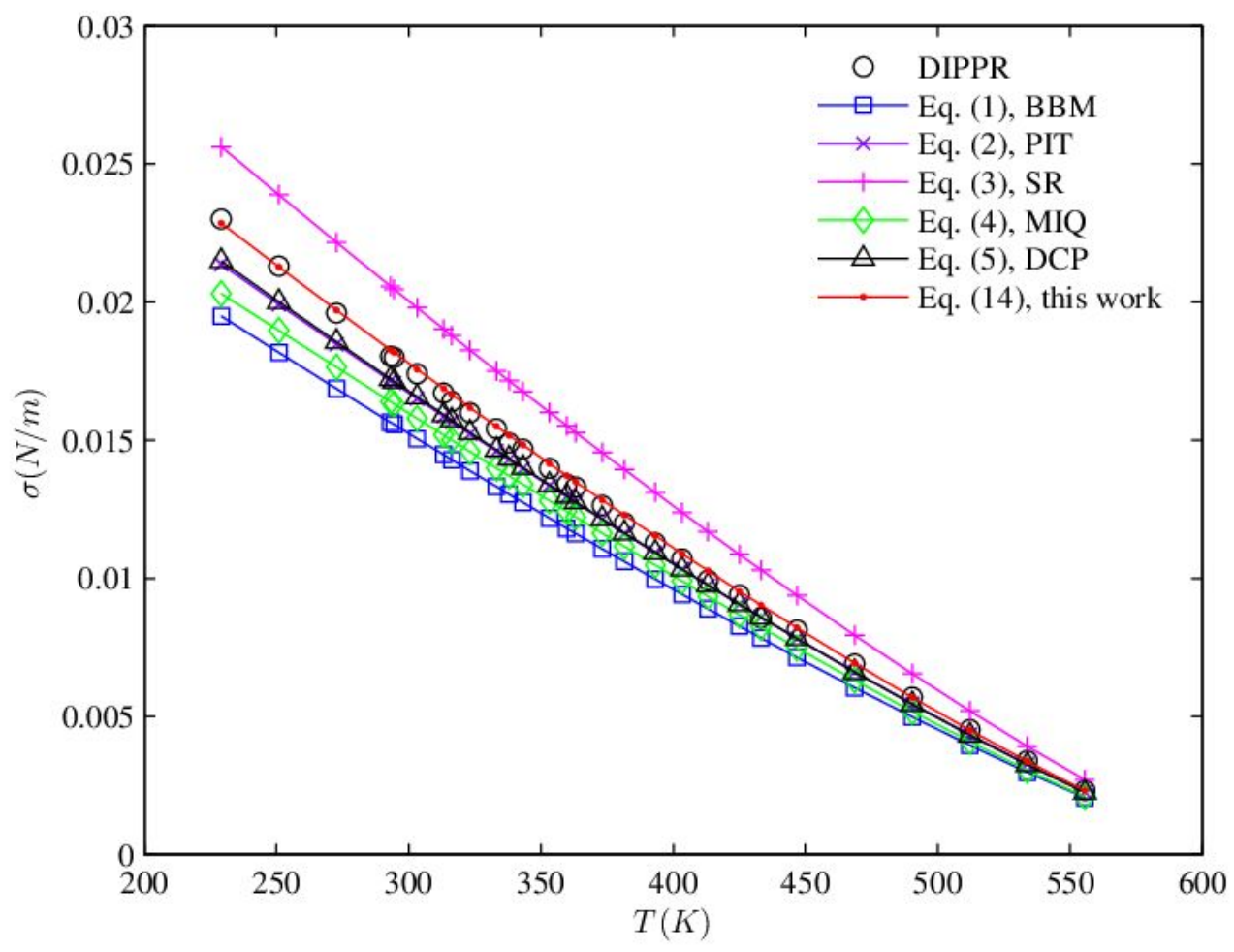

Fig. S1. Surface tension versus temperature for Decamethylcyclopentasiloxane. Data from DIPPR (points) and from different correlation models (lines).

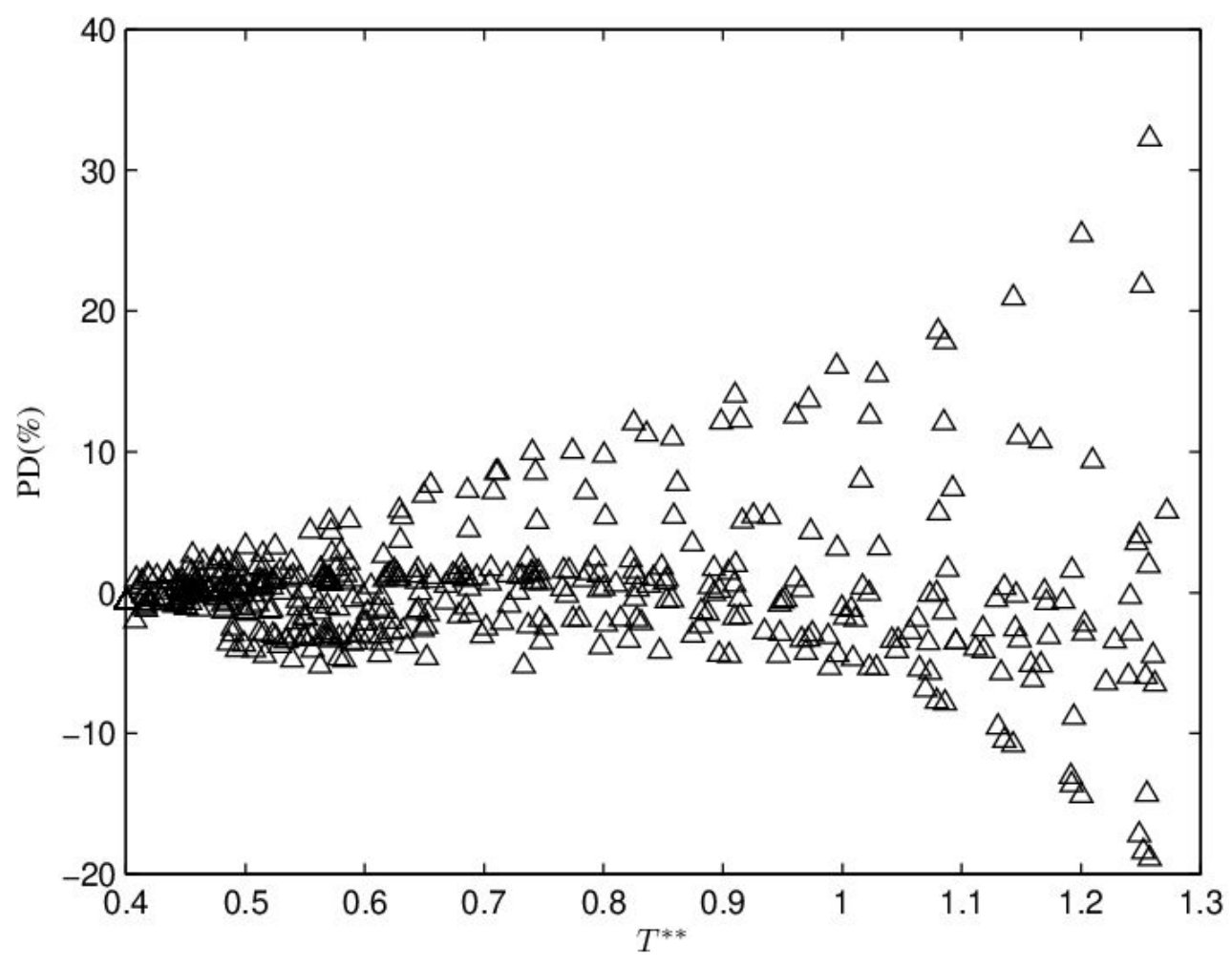

Fig. S2. Percentage deviations versus $T^{* *}$ for 484 data of 36 silanes 


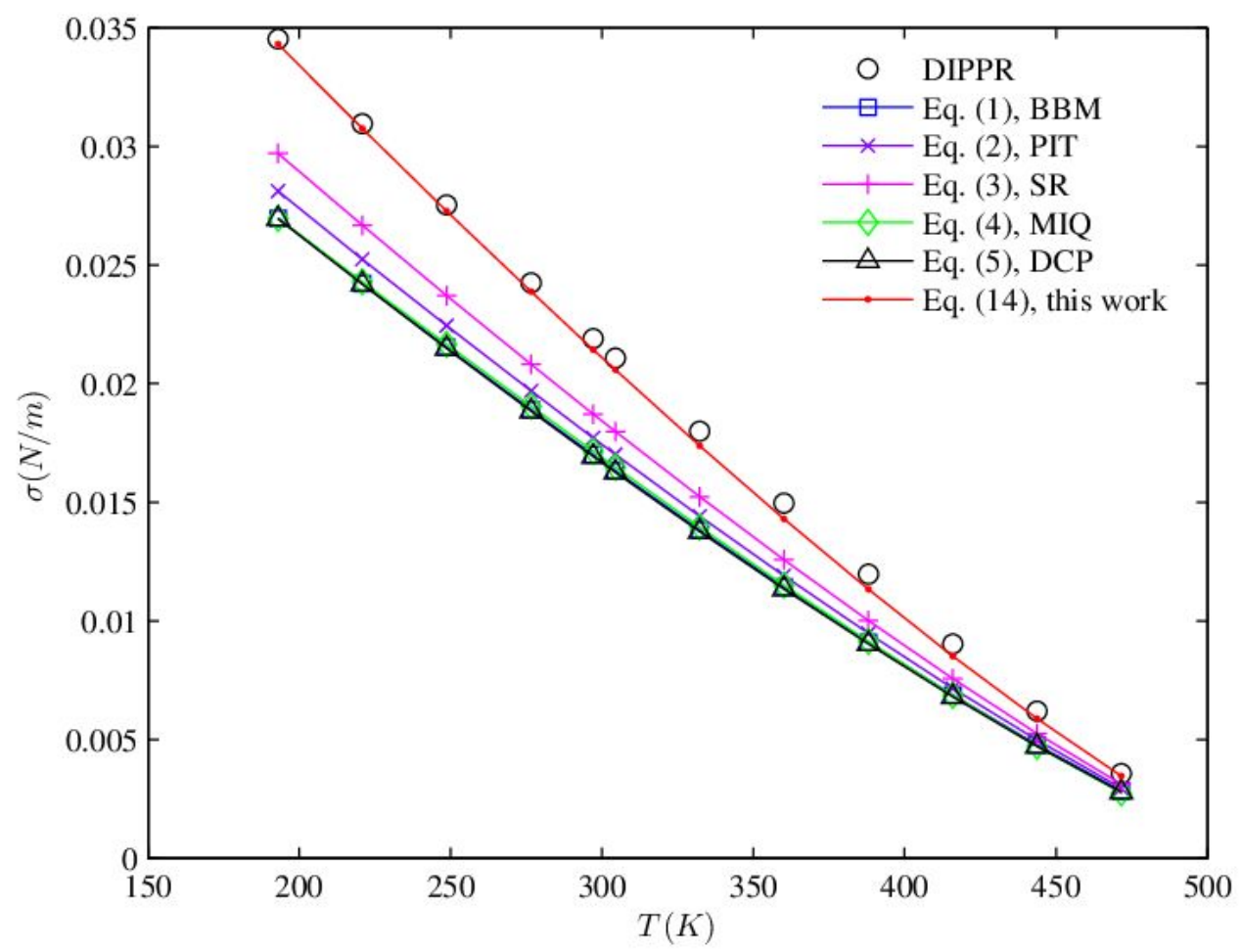

Fig. S3. Surface tension versus temperature for Dimethyldimethoxysilane. Data from DIPPR (points) and from different correlation models (lines).

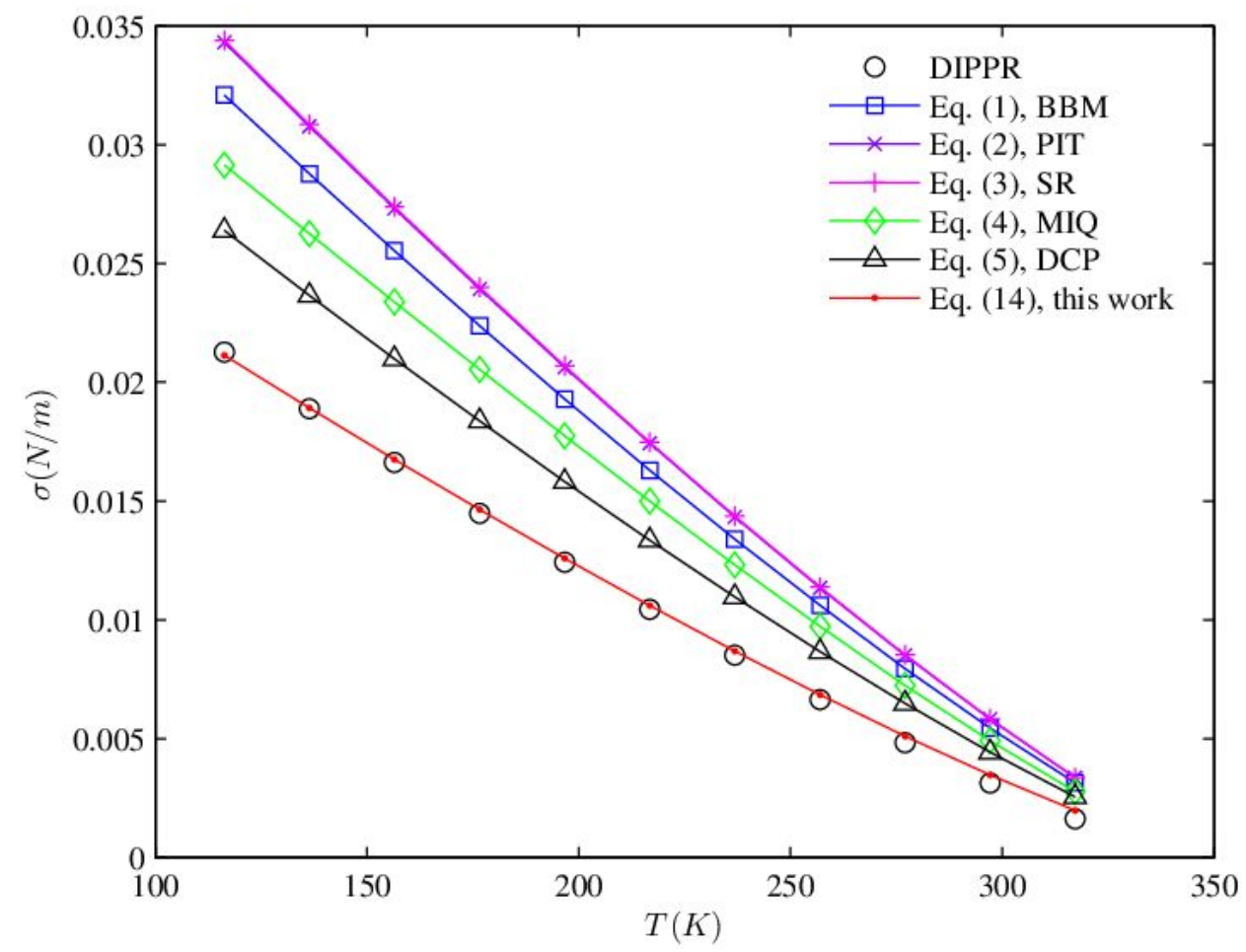

Fig. S4. Surface tension versus temperature for Methyl Silane. Data from DIPPR (points) and from different correlation models (lines). 


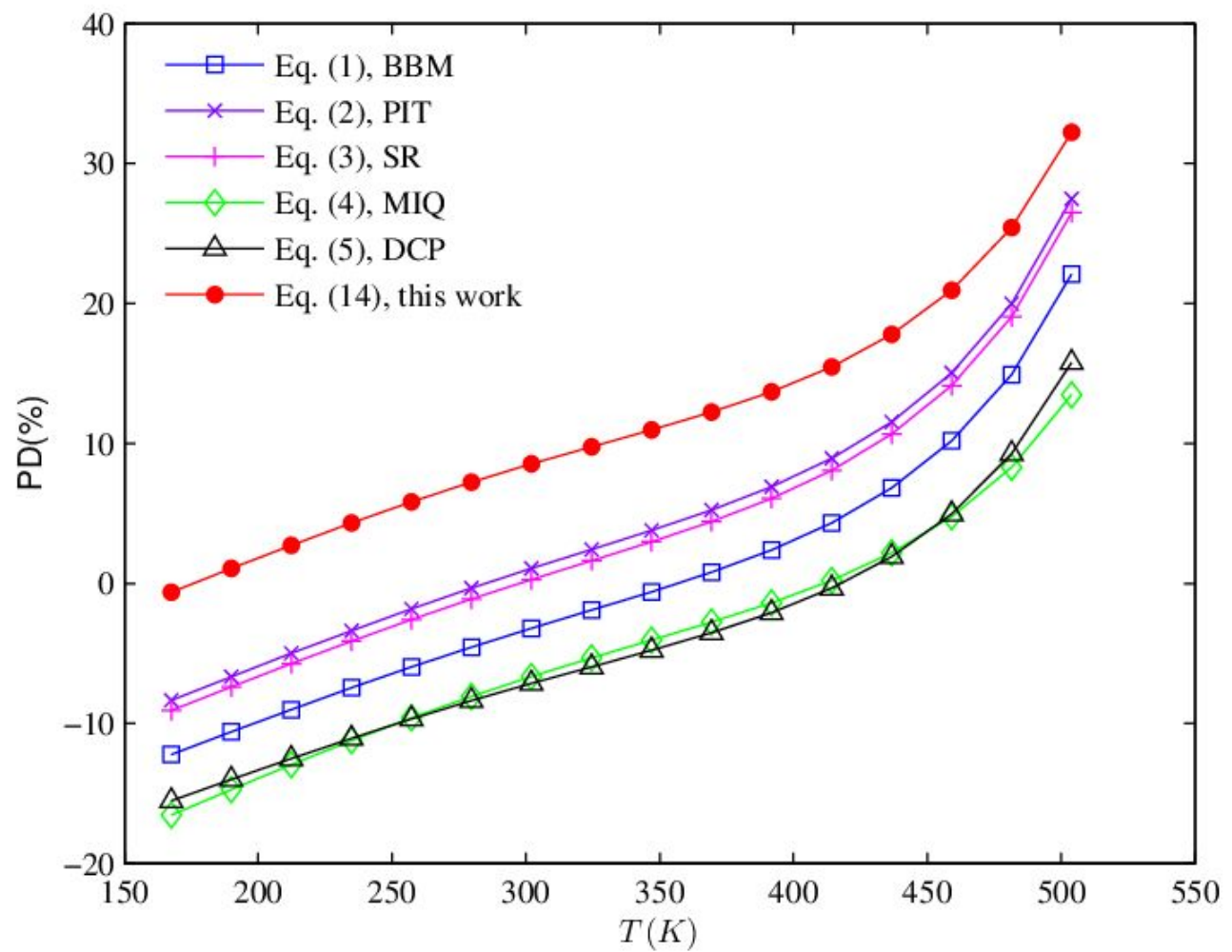

Fig. S5. Percentage deviations versus temperature for Ethyltrichlorosilane and each model. 